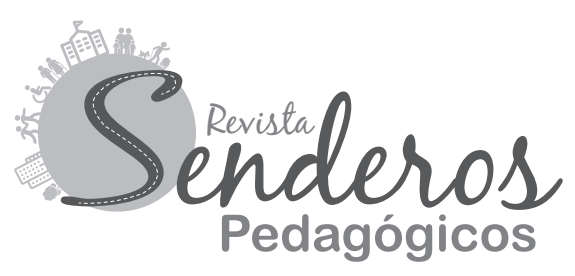

\title{
Aproximación antropológica a la metodología de la sistematización de experiencias ${ }^{1}$
}

\author{
Anthropological Approach to the Methodology \\ of the Systematization of Experiences
}

\author{
Autor: \\ Norling Sabel Solís Narváez ${ }^{2}$ \\ Recibido: 23/08/2019 \\ Aprobado: 14/11/2019
}

1. Artículo de reflexión no derivado de investigación. Fue presentado en el Congreso VII de Pensamiento Humanista Regional: El Humanismo en la sociedad contemporánea. 2017, bajo el título: El enfoque antropológico de la sistematización de experiencia en la construcción de conocimiento humanizado.

2. Universidad Nacional Autónoma de Nicaragua, UNANManagua, Docente investigador del Departamento de Antropología Editor Raíces: Revista Nicaragüense de Antropología. solisnorling@gmail.com

nsolis@unan.edu.ni

\section{Resumen}

Este artículo tiene como propósito promover la reflexión sobre el proceso metodológico de sistematización de experiencias, a partir de la capacitación teórico-práctica dirigida a miembros de la Cruz Roja Nicaragüense y Extensión Universitaria de la UNAN-Managua entre 2016 y 2018. Los talleres realizados tuvieron la finalidad de socializar los aspectos metodológicos de la sistematización de experiencias y poner en práctica los conocimientos adquiridos en sus contextos de trabajos. Los hallazgos obtenidos en este artículo son el resultado del análisis antropológico, y el método del reconocimiento cultural para la producción de conocimiento, durante las sesiones de capacitación. Evidenciando que la sistematización es, sin duda, uno de los procesos más humanizados de la producción de conocimiento. La sistematización como didáctica incursiona en la educación popular, como el proceso de enseñanzaaprendizaje que pone en el centro las experiencias y conocimiento acumulado por los protagonistas en entornos sociales y culturales. Los elementos antropológicos fueron imprescindibles para interpretar 
las subjetividades mediadas por el acervo cultural de los participantes. Se concluyó que la sistematización de experiencias es un recurso metodológico importante para las organizaciones, instituciones educativas y comunidades, con el objeto de extraer lecciones aprendidas que pueden ser aplicables a futuros procesos transformadores. La capacitación permitió visualizar fases metodológicas para lograr una interpretación profunda y crítica de las experiencias donde los sujetos son capaces de extraer las lecciones aprendidas surgidas en las sesiones de trabajo. Producto de la experiencia metodológica de capacitación se propuso un modelo para implementar el proceso de sistematización, que tiene las siguientes fases: a) definir objetivos y alcance de la sistematización; b) el punto de partida; c) fase de recolección documental -contraste de objetividades; d) fase de recolección oral; e) fase de reconstrucción de la experiencia; f) fase de análisis participativo -encuentro de subjetividades y, g) fase de interconexión crítica de consenso.

Palabras clave: sistematización, experiencia, metodología, humanismo, antropología.

\begin{abstract}
This article aims to promote reflection on the methodological process of systematization of experiences, based on theoreticalpractical training aimed at members of the Nicaraguan Red Cross and University Extension of UNAN-Managua between 2016 and 2018. The workshops held were the purpose of socializing the methodological aspects of the systematization of experiences and putting into practice the knowledge acquired in their work contexts. The findings
\end{abstract}

obtained in this article are the result of the anthropological analysis, and the method of cultural recognition for knowledge production, during training sessions. Proving that systematization is, without a doubt, one of the most humanized processes of knowledge production. Systematization as didactic ventures into popular education, as the teaching-learning process that puts the experiences and knowledge accumulated by the protagonists in social and cultural environments at the center. The anthropological elements were essential to interpret the subjectivities mediated by the cultural heritage of the participants. It was concluded that the systematization of experiences is an important methodological resource for organizations, educational institutions and communities, in order to extract lessons learned that may be applicable to future transformative processes. The training allowed us to visualize methodological phases to achieve a deep and critical interpretation of the experiences where the subjects are able to extract the lessons learned in the work sessions. As a result of the methodological training experience, a model was proposed to implement the systematization process, which has the following phases: a) define objectives and scope of systematization; $b$ ) the starting point; c) document collection phase - contrast of objectivities; d) oral collection phase; e) experience reconstruction phase; f) participatory analysis phase - meeting of subjectivities and, g) phase of critical consensus interconnection.

Keywords: Systematization, experience, methodology, humanism, anthropology. 


\section{Introducción}

Es una aventura plantear la sistematización de experiencias como metodología para el abordaje de lecciones aprendidas de programas o proyectos sociales. De acuerdo con Oscar Jara (2012) "Una de las dificultades más frecuentes que encontramos para poder precisar en qué consiste, específicamente, la sistematización de experiencias es la indefinición de las fronteras entre ella, la evaluación y la investigación social" (pág. 56).

La sistematización es aquella interpretación crítica de una o varias experiencias que, a partir de su ordenamiento y reconstrucción, permite descubrir o explicar la lógica del proceso vivido, los factores que han intervenido en dicho proceso, cómo se han relacionado entre sí, y por qué lo han hecho de ese modo (Holliday, 2003). La metodología de la sistematización de experiencias es propia de las experiencias de movimientos sociales y populares de América Latina (Holliday, Alforja - CEAAL, 2019).

Dicha interpretación lleva a un proceso metodológico para extraer lecciones aprendidas de las experiencias vividas. Las experiencias son procesos sociohistóricos dinámicos y complejos, individuales y colectivos que son vividas por personas concretas. No son simplemente hechos o acontecimientos puntuales, ni meramente datos. Las experiencias son esencialmente procesos vitales que están en permanente movimiento y combinan un conjunto de dimensiones objetivas y subjetivas de la realidad histórico-social (Holliday, Alforja - CEAAL, 2019)

La sistematización es una metodología que es poco utilizada en las ciencias sociales, en comparación con la evaluación e investigación social, pero que ha aumentado gradualmente su uso en la educación popular (Jara, 2012). La sistematización de experiencias es importante desde el punto de vista de la educación popular, ya que ha sido utilizada en procesos de aprendizajes colectivos y emancipadores como sostiene Jara (2012).

La sistematización de experiencias no es una investigación como tal, por tanto, no está sujeta a hipótesis previas. Analiza de manera crítica la experiencia de los sujetos con la intención de comprender su realidad social y cultural para transformarla (Acosta, 2005, pág. 8). La sistematización de experiencias es importante para el rescate de lecciones aprendidas que brinden conocimiento para mejorar futuras experiencias en el marco de una actividad, proyecto o programa de desarrollo comunitario. 
La sistematización de experiencias es un proceso metodológico comprendido por: a) definición de líneas, ejes y objetivos de la sistematización; b) el punto de partida; c) recuperación del proceso vivido; d) La reflexión de fondo y, e) los puntos de cierre (Jara, 2012). Como sostiene Jara (2017), no existe una definición clara de lo que es sistematización de experiencias, sin embargo, se fundamenta en un análisis crítico de la experiencia vivida para extraer lecciones aprendidas que sean de utilidad para los protagonistas de dicha experiencia, y para otros que lleven procesos similares, sin caer en el simple registro del pasado ni en un proceso evaluativo.

La propuesta de este artículo no parte de la sistematización de un programa o proyecto particular, sino, de un proceso de capacitación dirigido a un colectivo de docentes de la UNAN-Managua y Técnicos de la Cruz Roja Nicaragüense sobre la metodología de sistematización de experiencias que propone Oscar Jara (2012).

La sistematización de experiencias y la antropología ponen en el centro al ser humano, porque ambas se fundamentan sobre los hechos vividos y socializados de los mismos, según su contexto socio-histórico. La primera, documenta y reflexiona las vivencias de participantes en un programa, proyecto o actividad, para registrar lecciones aprendidas y transformadoras (Holliday, 2017, pág. 27) y, la segunda, estudia el comportamiento del ser humano en su modo de vida, cultura y tradición, de una forma holística e integral, a partir de las dinámicas de la sociedad (Escobar, 1999, pág. 6).

A partir de la implementación de la capacitación sobre cómo sistematizar experiencias, se aprendió a definir objetivos, ejes de sistematización, diseños de técnicas de entrevistas, observación y la interpretación crítica. La capacitación sobre sistematización de experiencias se llevó a cabo en varias etapas, entre ellas: explicación teórica-metodológica a través de talleres participativos, los cuales consistieron en crear un ambiente que generara la participación de todos los protagonistas desde sus experiencias, y asimilación del contenido. La siguiente etapa, la aplicación de lo aprendido en sus distintos contextos, es decir, una vez diseñada su propuesta, ejes y objetivos, regresaban a sus espacios para extraer la información necesaria.

Posteriormente, en la socialización del aprendizaje en los talleres participativos, donde cada subgrupo conformado con los participantes compartía su experiencia metodológica en la aplicación, se generaron aprendizajes colectivos. La última etapa de trabajo concluyó con un informe de sistematización de las experiencias vividas en sus contextos, los de la Cruz Roja aplicado al proyecto sobre Prevención del Zika y la UNAN-Managua, con la experiencia del programa Universidad en el Campo. 
Durante la aplicación de la metodología en sus contextos, se brindó el acompañamiento metodológico a los protagonistas y el ejercicio evidenció que la discusión giró en torno a los aspectos culturales, simbólicos y subjetivos que no se mencionan explícitamente en la propuesta de Jara (2012). Estas reflexiones se abordaron en plenarios colectivos, en donde surgieron nuevas ideas para interpretar las experiencias vividas que de forma individual no habían sido percibidas.

El análisis de las reflexiones de los participantes se realizó con una mirada antropológica, porque existen espacios en la memoria individual que se omiten cuando se está tratando de reconstruir una vivencia; así se observó durante los talleres de capacitación con los protagonistas de las instituciones mencionadas. Cuando se abordaron de forma colectiva fueron complementadas o se profundizó el análisis. El taller teórico-práctico consistió en apropiarse de la metodología y aplicarlo a su contexto de trabajo; por ejemplo, en el realizado con la Cruz Roja Nicaragüense, durante la etapa de reconstrucción de la experiencia y siguiendo la propuesta de Jara (2012), los protagonistas aprendieron a interpretar las reacciones de las personas cuando participan en procesos de sistematización. También, desarrollaron la mirada antropológica, es decir, la visión holística sobre la vivencia.

La sistematización de experiencias tiene sentido en tanto es una manera de producir conocimiento a partir de la cultura y el saber de las comunidades participantes (Holliday, 2017), porque son los protagonistas, los que a partir de la experiencia, tienen el conocimiento para generar nuevas ideas de transformación de su realidad (Geilfus, 2002); es decir, que las comunidades tienen conocimiento, pero al no ser sistematizado se pierde.

Por último, este trabajo está compuesto de tres secciones. La primera trata sobre la relación teórica-metodológica entre la antropología, la sistematización de experiencias y la educación popular. Esto con el propósito de analizar y entender los resultados. La segunda sección describe la utilidad de la antropología para comprender las experiencias vividas como procesos de interacción social. Es el resultado de la reflexión del autor producto del proceso de capacitación y acompañamiento a la Cruz Roja Nicaragüense y Extensión Universitaria de la UNAN-Managua entre el 2016 y 2018. Por último, en la tercera, se propone una variación metodológica que permita llevar a cabo una sistematización de experiencias, de manera complementaria a la propuesta metodológica de Oscar Jara (2012), con la incorporación del enfoque antropológico. La propuesta de sistematización con enfoque antropológico permite generar un conocimiento humanizado a partir del acervo cultural y el significado simbólico que tiene para 
los protagonistas, el cual pondrán de manifiesto al interpretar la experiencia vivida; esto permite que la realidad se analice en contexto, es decir, en el lugar donde vivió la experiencia como un escenario social donde los protagonistas son sujetos que se reconstruyen y definen entre sí, según su cultura.

\section{Simbiosis del conocimiento humanizado: antropología, sistematización de experiencias y educación popular}

La sistematización de experiencias como ruta metodológica para construir conocimiento empírico se ubica en el campo de la investigación cualitativa con enfoque crítico interpretativo, ya que busca descubrir e interpretar prácticas sociales singulares, privilegia el punto de vista de los actores, la subjetividad, la historia local y los sentidos en la práctica concreta (García \& Morgan Tirado, 2010).

La sistematización de experiencias se articula con la antropología social porque pone en el centro del análisis a la persona como sujeto partícipe y constructor de su realidad sociocultural, y se fundamenta en una experiencia intergeneracional; es decir, lo que la persona ha aprendido como transmisión oral y socialización dentro de la familia y la comunidad. Usa la educación popular como método de construcción de conocimiento porque privilegia la experiencia acumulada de sus protagonistas, como sostiene Paulo Freire (1972):

El conocimiento requiere de acción transformadora sobre la realidad y demanda una búsqueda constante; implica invención y reinvención. En el proceso de aprendizaje sólo aprende verdaderamente aquel que se apropia de lo aprendido, transformándolo en aprehendido con lo que puede por eso mismo reinventarlo: aquel que es capaz de aplicar lo aprendidoaprehendido a las situaciones concretas (p. 28).

La sistematización de experiencias retoma en palabras sencillas lo que las personas vivieron durante un periodo determinado en un programa, proyecto o actividad, para extraer las buenas prácticas, mejorar futuras experiencias propias o de terceros. Por ser la experiencia de alguien que tiene una carga cultural y se identifica con cierto grupo social, que además tiene sus propias conexiones e interpretaciones, se le da la posibilidad metodológica de que él mismo sea protagonista en la reconstrucción de su experiencia y pueda extraer lecciones aprendidas (Holliday, 2018)

Al darle voz a los protagonistas en los espacios de intercambio, reconstrucción e interpretación de la experiencia, se promueve el empoderamiento de las personas 
para transformar su propia realidad. Geilfus (2002) sugiere que "los beneficiarios" o "comunidad" estén ligados con los procesos de desarrollo comunitario que tienen efecto en su vida cotidiana, son ellos los que viven donde se ejecutará o ejecutó algún proyecto o programa.

Según Frans Geilfus “es un proceso mediante el cual la gente puede ganar más o menos grados de participación en el proceso de desarrollo” (p. 1). Por ello, en esta sistematización se tiene en cuenta al protagonista de la vivencia y se le brindan pautas para democratizar su participación durante la recopilación y reflexión de los datos ordenados en el proceso de sistematizar. Por tanto, la reflexión crítica de la experiencia podrá generar conocimiento sustancial para los actores locales, aunque la misma no tenga transcendencia más allá del grupo protagónico. Será útil para ellos, porque les permitirá visualizar de forma articulada y ordenada lo que vivieron.

Desde los primeros antropólogos se concibió la idea de que el conocimiento estaba en el "lugar”, en el "estar allí" (Geertz, 1992), por esa razón, en el proceso metodológico se entiende a la antropología como la ciencia que toma en cuenta el comportamiento del ser humano para generar conocimiento a partir de sus propias expresiones y estilos de vida. De esta manera, la sistematización de experiencias, que comprende volver a "lo vivido", reconstruye, analiza, cuestiona y reflexiona para extraer lecciones aprendidas (Velde, 2012); esto crea una relación armoniosa con la antropología para generar conocimiento de la experiencia vivida, al partir del acercamiento social y cultural.

Reconoce la forma en que cada persona vivió la experiencia, desde la antropología, el modo de ver y entender el universo de conocimiento de cada protagonista de la experiencia que está siendo sistematizada. Tanto la antropología como ciencia y la sistematización de experiencias como metodología, están íntimamente ligadas con la educación popular porque ambas ponen de manifiesto la experiencia situada, es decir, lo que el individuo ha acumulado como conocimiento durante un periodo de tiempo.

La antropología y la sistematización de experiencias se relacionan con la educación popular porque, esta última, permite socializar el conocimiento, crear condiciones de enseñanza-aprendizaje desde un enfoque participativo, al reconocer los aprendizajes previos de cada participante, enmarcados en un espacio socio-histórico, simbólico y cultural.

La educación popular es un proceso emancipador "no pretende ser teoría 'seca' porque constituye un sistema metodológico, necesariamente dinámico, cuyo 
objetivo consiste en facilitar la acción transformadora desde sectores populares para el mejoramiento de sus propias condiciones de vida” (Velde, 2012, pág. 1). Freire (1972) desde su experiencia infirió que el individuo en su seno familiar y comunal desarrolla un conocimiento que por sí mismo lo hace un ser educado, entendiendo lo educado, como el proceso de aprendizaje de vida y experiencia empírica que ha sido transmitida de generación en generación a través de réplica y de forma oral (p. 75).

Por ende, un sujeto que esté en un proceso educativo tiene una experiencia de vida acumulada que se vuelve parte de su conocimiento, y que además funciona para interpretar la realidad tal cual se socializa y aprehende en la cultura a la que está adscrito. La sistematización de experiencias extrae lecciones aprendidas de la experiencia vivida y la antropología interpreta el comportamiento de las personas en sus entornos de vida. Por tanto, son complementarias. A propósito de ello el antropólogo Claude Lévi-Strauss (1987) infiere que:

La antropología social no se encierra en una fracción del dominio de la etnología, no separa cultura material y cultura espiritual. En la perspectiva que le es propia - y que habremos de situar - les consagra igual atención. Los hombres se comunican por medio de símbolos y signos; para la antropología, que es una conversación del hombre con el hombre, todo es símbolo y signo que se afirma como intermediario entre dos objetos (p. 28).

El conocimiento como producto de la experiencia ha estado presente desde la existencia misma de la humanidad. Por tanto, si el ser humano avanza, el conocimiento aumenta. El conocimiento tiene su génesis en la experiencia empírica, en las vivencias y retos del individuo para adaptar la naturaleza día a día. Por tanto, la sistematización de experiencias es un proceso que conjuga la práctica empírica con la creación de conocimiento formal a partir de una vivencia o interacción social.

La educación popular genera conocimiento al hacer partícipes a los protagonistas en el proceso de formación, promueve la interacción social que se traduce en aprendizajes colectivos porque los participantes socializan lo aprendido, de manera que lo que sabe uno complementa lo que desconoce el otro. La participación de los protagonistas permitirá socializar los conocimientos que han sido aprendidos en sus entornos sociales, porque cada uno se vuelve un contenedor de conocimiento producto de otras socializaciones donde han interactuado como sujetos activos (Vygotsky, 1995). La antropología dice que la visión de lugar está conectada al sentido de pertenencia; por tanto, las experiencias que educan y 
generan conocimiento están ligadas con la cultura de la persona y su forma de entender el mundo (Godelier, 2010).

Las lecciones aprendidas se discuten con los involucrados en la práctica y se observa su comportamiento, haciendo uso de la etnografía aplicada, que es la descripción detallada de la conducta de un determinado grupo social y cultural, un retrato escrito de las poblaciones y comunidades (Guber, 2001). Se describe y se detalla la vinculación entre lo que dicen y hacen los protagonistas sobre su experiencia vivida. Se detallan las interacciones entre todos los actores internos y externos involucrados -que pueden tener intereses diversos e incluso contrapuestos- y, a la vez, se adapta la propuesta inicial a los cambios en el contexto de desarrollo (Barnechca y Morgan, 2010).

\section{Reconocimiento del "nos-otros" en las experiencias vividas y talleres de sistematización}

La metodología de sistematización de Oscar Jara fue el tema abordado en la capacitación con los miembros de la Cruz Roja Nicaragüense y de Extensión Universitaria de la UNAN-Managua -experiencia que fue el insumo para esta reflexión-.El objetivo principal fue compartir los conocimientos teóricos y metodológicos de la sistematización con estos actores, para que los mismos lo aplicaran en sus contextos.

Para desarrollar las capacitaciones se diseñó una propuesta de formación a través de los métodos participativos fundamentados en la propuesta de educación popular, apoyada en autores como Van de Velde (2012) y Paulo Freire (1972). La propuesta se implementó a través de encuentros formales en las salas de capacitación de la Cruz Roja, y en el Centro Ruso de la UNAN-Managua, en forma de talleres participativos.

Los talleres participativos según Geilfus (2002) son una estrategia de democratización de una investigación o diagnóstico, porque les permite a los participantes hacer uso de la palabra y generar interacción colectiva. Los talleres participativos se ejecutaron, pensando que la experiencia individual que cada persona había tenido al aplicar paso a paso la sistematización de experiencias, podría ser interpretada a través de la interacción del colectivo. 


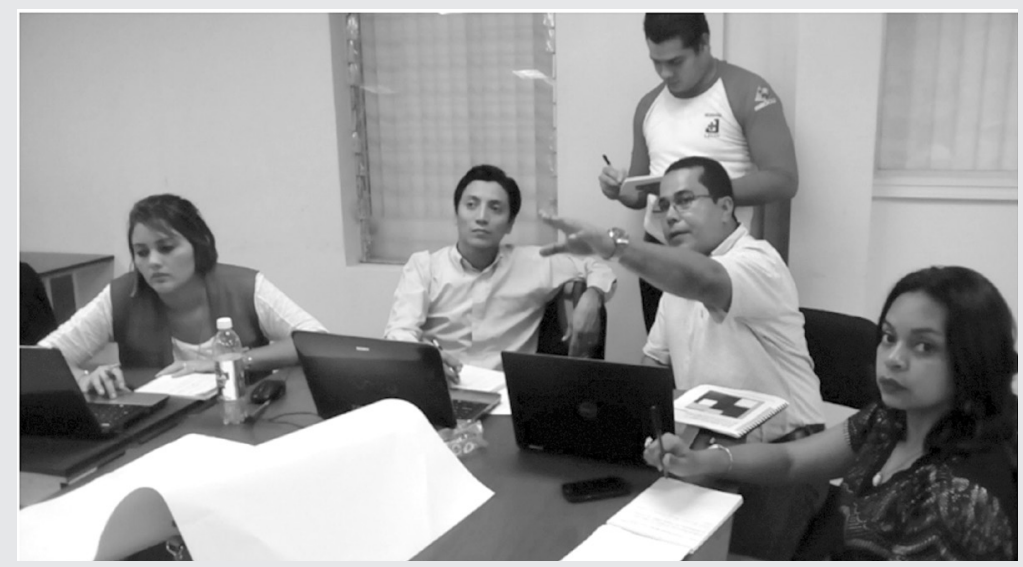

Figura 1. Técnicos de la Cruz Roja Nicaragüense, durante el proceso de aprendizaje de la metodología de sistematización de experiencias. 2016.

Autor fotografía: Norling Solís Narváez

Los talleres metodológicamente son una propuesta de aprendizaje para combinar el conocimiento formal con la experiencia vivida en etapas previas. Durante las sesiones de trabajo con los equipos antes mencionados, se identificó que las personas desarrollaron distintas formas de interpretación de la experiencia. Esto generó en el grupo, discusión y posterior consenso para lograr interpretaciones ajustadas a la realidad vivida. Muchas de estas interpretaciones se pudieron negociar por los protagonistas en el grupo, porque su acervo cultural les brindaba experiencias distintas, de acuerdo con el contexto donde lo vivieron.

Los talleres de sistematización de experiencias en los que participaron la Cruz Roja Nicaragüense y la UNAN-Managua, se llevaron a cabo en distintos momentos de reflexión y discusión sobre las experiencias que se estaban sistematizando. El facilitador fue un mediador en las discusiones que surgieron de las experiencias registradas. Los participantes tenían diversas posibilidades de entender cada experiencia, fenómeno, proyecto, programa a partir del diálogo entre lo empírico que habían vivido y la reflexión crítica.

Cada persona procesó la información de acuerdo con su manera de entenderla, por ejemplo, no fue lo mismo para una persona Garífuna de la Costa Caribe Nicaragüense interpretar su experiencia sobre el sistema de prevención del Zika (proyecto de la Cruz Roja) que para alguien que vive en Managua, en un barrio periférico. En ambos casos, fue primordial el reconocimiento del contexto en el que se desarrolló la experiencia, aunque fuera el mismo proyecto. 
Por eso, en la producción social del conocimiento se reconoce que el sistema cultural al que pertenece cada individuo, será el medio que filtre su interpretación de los eventos. Por tal razón, se deben sistematizar las experiencias de acuerdo con la situación en la que se dieron y según aquellos que participaron (Holliday, 2017).

Es importante reconocer que cuando las personas abordaron su experiencia, también vincularon las de los demás con la propia, a partir de una lógica de transferencia, para asociar su comentario con algo en lo que estaba de acuerdo con otro participante, o de contratransferencia para indicar algo en lo que no estaba de acuerdo.

La antropología lo entiende como una posibilidad de encuentro entre el YO y el OTRO (Kapuściński, 2007) lo que permitirá la posibilidad del "Nosotros" en un ambiente determinado. Los encuentros permiten que la experiencia sea entendida desde distintas ópticas, es decir, a partir de la mirada de quien la vivió. En la que la relación "yo" con el “otro” involucrado, generó un nos-otros en la experiencia (Krotz, 1994), ese otro, pueden ser tanto los participantes directos como agentes externos indirectos.

Los talleres fueron una estrategia didáctica para desarrollar la capacitación y la experiencia de compartir conocimiento permitió evidenciar la forma en que conectó la experiencia de los participantes con lo que se explicó en las sesiones de trabajo. Se propusieron dos formas de interpretar la experiencia vivida, a partir de lo que los participantes manifestaban en los talleres.

Primero, un diálogo interno entre la persona y su experiencia, es decir, aquellos elementos que surgen inconscientemente y que están alojados en su subconsciente, producto de su pertenencia cultural. Segundo, un diálogo externo entre la persona, su experiencia y los otros actores involucrados En este sentido, la interpretación sobre la experiencia fue más consciente pero medida, porque convergió con la experiencia que tuvieron las otras personas en el mismo contexto. Por ejemplo, para el primer caso, a una persona que estaba acostumbrada a levantarse de madrugada y cocinar en fogón (cocina de leña), se le hizo partícipe de un proyecto de cocina solar, pero su experiencia inconsciente la llevará a intentar usar la cocina en los momentos en que culturalmente lo ha aprendido, en la madrugada; en el momento que tenga que compartir su experiencia externamente, de forma consciente será distinta que las de otros, por tanto la interpretación reflejará diferencias objetivas y subjetivas. 
Solis, $N$.

Esteban Krotz, (1994) indica que:

Un ser humano reconocido en el sentido descrito como otro no es considerado con respecto a sus particularidades altamente individuales y mucho menos con respecto a sus propiedades "naturales" como tal, sino como miembro de una sociedad, como portador de una cultura, como heredero de una tradición, como representante de una colectividad, como nudo de una estructura comunicativa de larga duración, como iniciado en un universo simbólico, como introducido a una forma de vida diferente de otras - todo esto significa también, como resultado y creador partícipe de un proceso histórico específico, único e irrepetible (p. 9).

Cada persona tendrá una manera distinta de comprender la experiencia; sin embargo, es válida para realizar la interpretación de la misma y extraer lecciones aprendidas. El conocimiento producido no es neutro, ni los valores que se le dan al aprendizaje son aplicables y utilizables de la misma forma en cualquier parte, inclusive fuera de la comunidad, puesto que, lo que se necesita extraer son lecciones aprendidas y no recetas (Van de Velde, 2008).

La construcción social del conocimiento permite que cada comunidad se apropie de su experiencia y elabore sus propias interpretaciones. Las interpretaciones de cada persona también deberán ser analizadas en el contexto donde ocurrió o se vivió la experiencia. Deberán ser reflexionadas desde y con los protagonistas, reconocer la importancia de sus aportes al análisis crítico. El facilitador y los participantes de la sistematización de experiencias podrán realizar etnografía del contexto donde se desenvuelven durante los intercambios grupales de reflexión, esto brindará mayores insumos para crear conocimientos.

Aquí se propone hacer etnografía de la propia experiencia, esto permite que los participantes realicen un retrato escrito de lo que vivieron, cómo lo vivieron y por qué; vinculado con su espiritualidad, relaciones de género, relaciones sociales, entre otros elementos necesarios de registrar. Las personas se vuelven autores de sus propias historias, relatan lo que vieron y escucharon. Les permite asociar sus experiencias con lo que observaron de otras personas que compartían el mismo espacio. Ese conocimiento construido colectivamente lleva implícita una propuesta consensuada de quehacer, lo que permite, a su vez, convertir ese conocimiento compartido en el mejor escenario para generar estrategias de mejora, desarrollo comunitario, desarrollo social a partir de las lecciones aprendidas (Velde, 2008). 


\section{Antropología y sistematización: encuentro de subjetividades, y contrastes de objetividades}

Las experiencias son procesos históricos y sociales dinámicos: están en permanente cambio y movimiento (Holliday, 2012). La sistematización de experiencias es una propuesta de reflexión y construcción de conocimiento humanizado, es decir, respeta y reconoce la experiencia vivida en cualquier espacio sin catalogarse de buena o mala para los participantes.

Las experiencias son procesos complejos porque en ellas intervienen una serie de factores objetivos y subjetivos que están en interrelación. Las condiciones de contexto o momento histórico en que se desenvuelven los protagonistas con sus experiencias son una fuente misma de conocimiento; por tanto, la interpretación y relación de los elementos vinculados con la experiencia tienen la posibilidad de generar información para quienes conducen el proceso de sistematización, es decir, el equipo encargado de sistematizar podrá utilizar como recurso de análisis: el contexto de origen, las características sociales, los códigos culturales y su forma de entender la experiencia para obtener una interpretación reflexiva que genere lecciones aprendidas.

Las personas que viven una experiencia, tienen de ella, una construcción simbólica, es decir, situaciones significativas que les permitirán asociarla con momentos particulares, palabras, o relaciones sociales. Así mismo, tienen elementos observables que son la memoria física de lo que vivieron, por ejemplo, para una persona que estudió la carrera de Desarrollo rural con el programa Universidad en el Campo (UNICAM) de la UNAN-Managua, los símbolos subjetivos para interpretar son: las relaciones con sus compañeros, su experiencia con los conocimientos técnicos, alguna materia que se le dificultó y le dejó una experiencia particular y, sus elementos observables, podrían ser: el cuaderno, el acta de calificaciones, la mochila, o cualquier otro objeto que le recuerde su experiencia en el aula de clases. Todo lo que el sujeto recuerde será vinculante entre lo que se aloja en su subjetividad y lo que evidencia su experiencia materializada.

Esos elementos son detectables en los discursos de los protagonistas, e incluso en la reconstrucción de la experiencia a partir de las fuentes documentales que tenga el equipo sistematizador. Sin embargo, las personas tienen percepciones, interpretaciones, intuiciones y emociones asociadas a los momentos que vivieron. Estos, transforman su realidad de forma que cualquier experiencia la adaptarían con su lógica de entender el mundo y, las relaciones que se establecen entre las personas, son piezas claves de la experiencia por reconstruir. 
Alfredo Ghiso (1998), reconoce en su propuesta de sistematización algunas miradas para desarrollarla: histórico-dialéctico, dialógico e interactivo, hermenéutico, reflexibilidad y deconstructivo. A continuación, se describen las tres miradas sobre la sistematización de experiencias.

En primer lugar y, como un proceso histórico - dialéctico, las experiencias son parte de una práctica social común y cotidiana; diariamente las personas se ven en la necesidad de reflexionar e interpretar un acontecimiento histórico que vivieron para plantear estrategias de mejora. La misma experiencia al ser social y vinculada con la cultura se vuelve dialéctica, es decir, se dan contradicciones internas en el individuo sobre cómo quiso vivir su experiencia y cómo la vivió; también existen contradicciones sobre la planificación de las actividades que generan experiencias, por ejemplo, cuestionar la experiencia según los resultados obtenidos, porque la planificación no resultó como tal. Se habla entonces de una experiencia con posibilidades de leerse de distintas formas, desde cómo se planificó, como se ejecutó, cómo se quería vivir y cómo se vivió, lo cual genera distintas subjetividades en los participantes (Ghiso, 1998).

Segundo, la sistematización de experiencias, al ser dialógica e interactiva, se construye como espacios de interacción, comunicación y relación, en los cuales se pueden interpretar el lenguaje utilizado por los involucrados y las relaciones contextualizadas en el espacio en que se desarrolló la experiencia (Ghiso, 1998). Desde este enfoque, cada participante durante el proceso de sistematización tiene una percepción e interpretación de la experiencia desde su perspectiva, dichas interpretaciones deben ser analizadas y reflexionadas para tener un panorama más amplio e integral de la experiencia. Cuando estás subjetividades se encuentran, el facilitador tiene un universo amplio de la experiencia, esto le permite generar mayores recursos de análisis y posibles lecciones aprendidas para ser comunicadas.

Desde una mirada hermenéutica, la sistematización de experiencias (Ghiso, 1998), retoma la antropología y su interpretación de signos, símbolos y elementos significantes que comprende la asociación de símbolos entre quienes vivieron la experiencia. La antropología debe interpretar en profundidad la cultura de las personas, dando significados a los elementos que representan "algo" para ellas; es decir, las experiencias de proyectos y programas están ligadas a elementos simbólicos que se vinculan con la realidad social, cultural, económica y política de los protagonistas (Geertz, 1992).

Cuando se contrasta lo objetivo con lo subjetivo, puede que existan elementos asociados que dan pautas de interpretación más profunda a la experiencia de una 
persona. Se retoma nuevamente el ejemplo de las cocinas solares. Sistematizar la experiencia de las personas de comunidades rurales en un proyecto de cocinas solares puede generar contraste entre la objetividad del proyecto que se centra en erradicar el uso del fogón, y los elementos subjetivos inmersos en las prácticas culturales de las personas participantes, que van a desarrollar la experiencia desde sus características socioculturales y, no necesariamente, como estaba planificado en los objetivos del proyecto inicial. Las personas en el campo de Nicaragua se levantan a cocinar a las 4 o 5 de la mañana en muchos departamentos, para que los trabajadores se lleven la comida a las parcelas, por ello, cuando el sol sale, la comida ya está lista. Quizás el informe de los evaluadores del proyecto sostendrá que tuvo resultados negativos porque las personas no usaron la cocina, y podrían añadir que esto ocurrió por la falta de conocimiento e interés. Pero, si se sistematiza la experiencia y se visualizan los elementos culturales y sociales que la llevaron a situaciones no planificadas, se podrían obtener lecciones aprendidas para otros proyectos similares que no tomaron en cuenta la vida cotidiana y cultura de la población.

En el escenario descrito sobre el uso de las cocinas solares, las preguntas de reflexión para extraer lecciones aprendidas en la sistematización de la experiencia de los participantes serían: ¿por qué las personas no usaron la cocina solar? ¿Qué aspectos culturales asociados a su experiencia cotidiana dirigieron el uso de la cocina a otra dirección? a ello, se le agregaría la etnografía del contexto, para describir los elementos culturales evidenciados durante la experiencia del proyecto de la cocina solar. La interpretación antropológica para decodificar los símbolos subjetivos que permitieron que la experiencia de la persona no fuera, según la esperada en el proyecto de cocina solar; por tanto, brindaría insumos para el reconocimiento de la cultura local antes de la implementación de un proyecto de este tipo.

La antropología involucrada en la sistematización permite interpretar todos los elementos vinculantes de forma crítica, extrae lecciones aprendidas reales, en el ámbito -social, cultural, económico, etc.- La sistematización de experiencias comprende además una reflexividad permanente en el proceso metodológico porque permite regresar a etapas previas, redirigir las técnicas de recolección de información: observación y entrevista (Guber, 2001).

Así, se infieren en el interior de las comunidades lecciones aprendidas en la interacción constante. No se reduce a narrar acontecimientos, describir procesos, escribir una memoria, clasificar tipos de experiencias, ordenar los datos, sino al fundamento de una interpretación crítica de la experiencia vivida, asociándola a los elementos simbólicos que se dieron en el espacio-tiempo de dicha experiencia. 


\section{Acercamiento a una propuesta antropológica de la sistematización de experiencia}

Es necesario indicar que la propuesta que en este artículo se presenta, es reflexiva y metodológica, parte del proceso de capacitación sobre la metodología de Oscar Jara (2012), discutida y analizada en los talleres con la Cruz Roja Nicaragüense y la UNAN-Managua. Sin embargo, las mismas propuestas, son solo eso, propuestas. Estas pueden variar según el contexto, participantes y ambiente social, cultural, político, etc. en el que se dio dicha experiencia.

Para entender la propuesta que suscita la experiencia de capacitación ya mencionada, se enuncia la metodología caracol de Jara (2012). a) Definición de líneas, ejes y objetivos de la sistematización, b) punto de partida, c) Recuperación del proceso vivido, d) reflexión de fondo, e) puntos de cierre.

Los talleres de capacitación sobre sistematización de experiencias dirigidos a la UNAN-Managua y a la Cruz Roja Nicaragüense, permitieron reconstruir una propuesta con mirada antropológica, dada la inclusión del componente etnográfico y la interpretación simbólica. Es importante para la sistematización de experiencias diseñar una guía metodológica que incluya técnicas de recolección de información y procesamiento de datos. Ello implica que una vez que se haya levantado la información de campo, los datos permitirán extraer lo sustancial para interpretarlos. Las fases propuestas a continuación tienen elementos originales incorporados por el autor de este artículo.

a) Definir objetivos y alcance de la sistematización: siguiendo la propuesta de Jara (2012), se procura definir, tiempo, espacio, actores, y alcance de la sistematización. Para ello será necesario tener un acercamiento a la experiencia para lograr determinar objetivos precisos.

b) El punto de partida: Según Jara (2012) solo pueden sistematizar la experiencia quienes participaron de la misma. Mientras no se tenga participación directa de la experiencia, la opinión y reflexión estarán sujetas a datos objetivos del informe y, por lo tanto, carentes de la interpretación subjetiva de los participantes.

c) Fase de recolección documental -contraste de objetividades-: Esta surge de la experiencia metodológica vivida con la aplicación de los talleres de capacitación a las instituciones mencionadas en este artículo. Lo que indica realizar una búsqueda y clasificación documental y bibliográfica 
de la experiencia. Son válidos los informes, la evaluación del programa, informe de seguimiento, memorias, actas o cualquier documento escrito que permita reconstruir la experiencia, pero además, apoye el análisis.

Esta fase es la que permitirá el contraste de la objetividad con la experiencia misma, es decir, entre lo que se planificó con lo que se vivió. Para lograr esta fase, es importante tener un equipo de trabajo, que además de recepcionar los documentos, clasifique, ordene y direccione estrategias metodológicas a seguir. Por ejemplo, indicar a partir de la documentación quienes serían informantes claves, o se pueden contemplar para encontrar informaciones especiales.

d) Fase de recolección oral: esta fase pertenece al momento de reconstrucción; en ella se preparan los instrumentos según la técnica a utilizar para la recolección de la información, fundamentalmente, las percepciones. En este sentido, se sugiere utilizar las técnicas observación, entrevistas y grupos focales; la primera y la segunda, permiten tener un panorama de las percepciones individuales de quienes participaron de la experiencia y, la tercera, maneja una percepción colectiva de los actores involucrados en la experiencia.

La técnica de entrevista en el método etnográfico de la antropología debe contemplar preguntas abiertas y semi estructuradas que generen la amplitud en las respuestas (Guber, 2001). También se hará uso de la observación participante durante los grupos focales, el facilitador debe tener dominio de los detalles para poder realizar preguntas reflexivas y observar el comportamiento y reacciones.

Para cumplir con esta fase es necesario que el equipo de trabajo responsable prepare una metodología con visión reflexiva, es decir, no esquematizada, sino que pueda regresar y rediseñarse si fuese necesario. Con el uso de la observación directa o participante -si el proyecto está en función aúnpuede generar apuntes que vinculen el discurso con las acciones. Como es de experiencias vividas podría el equipo de trabajo detallar en el Diario de campo, elementos que son observables y que funcionan para la reflexión. El dato que surja de las notas de campo se utilizará para la extracción de las lecciones aprendidas.

e) Fase de reconstrucción de la experiencia: la fase de reconstrucción de la información permitirá que el equipo encargado de recolectar la 
información procese los datos y reconstruya la experiencia, la cual evidenciará los éxitos y las fallas en el proceso. Se prepara un informe y se extraen los éxitos y las fallas, por aparte, para su análisis. Esta etapa solo se reconstruye con la participación de los protagonistas, pero conducida por el equipo de trabajo. El equipo sistematizador se puede apoyar en lo encontrado en los documentos y el discurso de los protagonistas, además de que se les solicita a estos etnografiar sus experiencias como recurso complementario.

Esto por supuesto debe estar íntimamente ligado con los elementos que las personas vinculan a la experiencia. También se debe realizar la interconexión de punto en común en el discurso como parte de la construcción en colectivo, por ejemplo, regresando al proyecto de cocina, si las personas indicaran en sus discursos que ellos acostumbran a cocinar en la madrugada para las fincas, la acción es común para todos los protagonistas. Reflexionar en el colectivo, intentando apoyarse en las notas del diario y observaciones etnográficas del comportamiento.

f) Fase de análisis participativo -encuentro de subjetividades-: teniendo la experiencia reconstruida con los éxitos y las fallas en el proceso, se realiza un taller participativo, en el cual los participantes de la experiencia y, preferiblemente, quienes brindaron la información analicen por qué y cómo sucedieron los hechos narrados, cómo se logró el éxito y por qué se falló, y qué se propone para mejorar. En esta etapa queda claro que los participantes entrarán en una fase de análisis de cada componente de éxito y fracaso que permitirá posteriormente obtener las lecciones aprendidas. En esta fase, se recomienda utilizar estrategias de proyección, es decir, que los participantes cuestionen cómo sería si hubiese realizado la actividad tal cual fue planificada, o como sería la actividad con las adaptaciones sugeridas por los participantes.

g) Fase de interconexión crítica de consenso: esta fase comprende la última en el proceso metodológico propuesto en este artículo. En ella se afinan las reflexiones de los elementos brindados por los participantes del taller, para extraer las lecciones aprendidas, y transformar la experiencia en una herramienta para mejorar futuros procesos de proyectos y programas. Interconexión crítica de consenso implica que, en las opiniones, reflexiones y discusiones, existen elementos en común, los cuales pueden extraerse y, a través de relación de testimonios de los participantes, pueden construir definiciones o propuestas concretas. La interpretación crítica debe suponer 
la vinculación de los elementos objetivamente verificables, por ejemplo, el diseño inicial del proyecto, fotografías, entre otros; además de los símbolos sociales y culturales subjetivos asociados a lo vivido. Debe entrar en punto de análisis la interrelación de los protagonistas y el contexto en el que se desarrolló la experiencia.

Se sostiene que esta propuesta, es solo eso, una propuesta. Que bien puede ser repensada, cuestionada y reconstruida, con el fin de que cada realidad construya su propio modelo de análisis. Aquí se muestra esta propuesta metodológica con el uso de la antropología, sujeta a construcciones permanentes.

\section{Conclusiones}

Las disciplinas sociales y humanísticas por su orientación científica están en busca de renovar, reformular, argumentar o bien crear nuevos conocimientos desde sus herramientas, técnicas, métodos y teorías. Sin embargo, hay un punto en común entre cada una de las disciplinas, no existe una en particular, que responda de forma íntegra y global a un fenómeno. La sistematización de experiencias como metodología participativa permite que los protagonistas se autoreconozcan dentro de una experiencia vivida, analizando y reflexionando sobre las condiciones sociales y culturales, vinculadas al cumplimiento de dicho momento vivido.

Ese enfoque participativo permite la conexión directa entre la antropología y la sistematización, puesto que con la primera se logra comprender el comportamiento de una persona desde su experiencia social-cultural y, la segunda, deja cuestionar y extraer de dicha experiencia lecciones aprendidas aplicables a futuras experiencias. La antropología que permite desde el método etnográfico la aplicación de técnicas como la observación y la entrevista, es solo una de las estrategias para generar conocimiento. Aquí se expresa constantemente que es necesario el desarrollo de talleres participativos desde la educación popular, para que los protagonistas puedan generar conocimientos transformadores de su realidad.

Desde los talleres participativos dirigidos a la Cruz Roja Nicaragüense y la UNAN-Managua, se logra redimensionar la importancia de contextualizar la experiencia, de enfocar las posturas individuales en contrastes con las posturas en colectivo, de coaccionar puntos de común denominador en los discursos donde los protagonistas no hablan de "su" experiencia, sino de "nuestra" experiencia. Por tanto, se reconoce el ámbito social de las experiencias, la 
incorporación del enfoque antropológico, para realizar el encuentro de las subjetividades de cada participante.

Por otro lado, es importante indicar que la experiencia vivida también genera símbolos en el proceso, estos pueden ser materiales, es decir, vincular ciertos elementos visibles al éxito o fracaso de una experiencia; o bien pueden ser subjetivos, inmateriales, es decir, que entre los discursos se generen matices con significaciones, que solo ese colectivo comprende, y que cada vez que se refieran a eso podrán asociarlo con la experiencia vivida.

Por último, es importante considerar que dentro de cualquier propuesta de sistematización la participación directa de los protagonistas se vuelve tan necesaria como pertinente. Y que además, metodológicamente, se propone en este trabajo agregarle un elemento más: la fase de análisis participativo. De manera que, la reflexión no quede en manos solo de quien sistematiza, sino también de los mismos protagonistas, que discutan y reflexionen su propia experiencia y generen un conocimiento humanizado que sustancialmente se vuelve coherente y pertinente a esa realidad en la que se vivió la experiencia.

\section{Referencias}

Acosta, L. A. (2005). Guía práctica para la sistematización de proyectos y programas de cooperación técnica. Oficina Regional de la FAO para América Latina y el Caribe.

Aguilar, J. (2011). Conocimiento libre y educación enmancipadora. Educare, 84-106.

Barnechea, M. M., \& Morgan Tirado, M. (2010). La sistematización de experiencias: producción de conocimientos desde y para la práctica. Tendencias.

Deslauriers, J. (2004). Investigación Cualitativa . Pereira: Editorial Papiro .

Eizagirre, M., \& Zabala, N. (7 de Agosto de 2006). Diccionario de Acción Humanitaria. Obtenido de http://www.dicc.hegoa.ehu.es/listar/mostrar/132

Escobar, A. (1999). Cultura, ambiente y política en la antropología contemporánea.

Primera Edición. Colombia: Instituto Colombiano de Antropología Ministerio de Cultura .

Freire, P. (1968). Una educación para la liberación. Argentina: Siglo XXI.

Freire, P. (1972). Pedagogía del oprimido. Buenos Aires: Siglo XXI. 
García, M. M., \& Morgan Tirado, M. (2010). La sistematización de experiencias: producción de conocimientos desde y para la práctica. Revista Tend. Retos, 97-108.

Geertz, C. (1992). La interpretación de las culturas . Barcelona: Gedisa.

Geilfus, F. (2002). Las 80 Herramientas para el desarrollo participativo. San Jose : IICA.

Ghiso, A. (1998). De la práctica singular al dialógo con lo plural: aproximaciones a otros transitos y sentidos de la sistematización en épocas de globalización. CEAAL.

Godelier, M. ( 2010). Comunidad, sociedad, cultura. Tres claves para comprender las identidades en conflicto. . Cuadernos de Antropología Social, 32, 13-29.

Guber, R. (2001). La etnografía: Método, campo y reflexividad. . Barcelona: Norma.

Holliday, O. J. (2003). ¿Qué es sistematizar? En G. Bustillos, Para sistematizar experiencias. Selección de lecturas sobre sistematización. . La Habana: CIE.

Holliday, O. J. (2012). Sistematización de experiencia, investigación y evaluación: aproximaciones desde tres ángulos. Educación Global, 56-71.

Holliday, O. J. (2017). La sistematización de experiencias: práctica y teoría para otros mundos posibles. Edición 2017. Bogotá: CINDE.

Holliday, O. J. (2018). La sistematización de experiencias: práctica y teoría para otros mundos políticos - 1ed. Bogotá: Centro Internacional de Educación y Desarrollo Humano - CINDE.

Holliday, O. J. (20 de Octubre de 2019). Alforja - CEAAL. Obtenido de La sistematización de experiencias y las corrientes innovadoras del pensamiento Latinoamericano- una aproximación histórica : http:// www.cepalforja.org/sistem/sistem_old/oscar_jara-sistematizacion_y_ corrientes_innovadoras.pdf

Kapila, S., \& Moher, R. (1995). Disciplinas interactivas: Principios para la investigacion interdisciplinaria. Otawa: International Development Research Centre Policy and Planning Group.

Kapuściński, R. (2007). El encuentro con el otro. España: Anagrama. Krotz, E. (1994). Alteridad y pregunta antropológica. Alteridades, 5-11.

Lévi-Strauss, C. (1987). Antropología estructural . Barcelona: Paidós. 
Narváez, N. S. (2015). Hacia la investigación multidisciplinar e interdisciplinar: reflexión sobre la aplicación de metodologías participativas y la Investigación Acción Aarticipativa . Humanismo y Cambio Social, 5, 74-82.

Sampieri, R. H., Fernandez Collado, C., \& Baptista Lucio, M. (2010). Metodologia de la investigacion. Quinta edicion. Mexico: Interamericana Editores.

Velde, H. V. (2008). Sistematización de experiencia. Texto de consulta. Estelí: Centro de Investigación, Capacitación y Acción Pedagógica (CICAP)/ Volens Centroamérica. Colección: Cuadernos del Desarrollo Comunitario.

Velde, H. V. (2012). Sistematización de experiencias: Esencia de una educación popular. . Obtenido de ABACO en Red: http://abacoenred.com/

Vygotsky, L. S. (1995). Pensamiento y lenguaje. Teoría del desarrollo cultural de las funciones psíquicas. Ediciones Fausto. 